\title{
An Environment for Contrast-Based Treatment of Amblyopia Using 3D Technology
}

\author{
Andrea Vitali, Giancarlo Facoetti, Angelo Gargantini \\ Department of Engineering, University of Bergamo \\ Viale Marconi 524044 - Dalmine (BG), ITALY \\ andrea.vitali1@unibg.it, giancarlo.facoetti@unibg.it, angelo.gargantini@unibg.it
}

\begin{abstract}
We have developed an application based on binocular vision for amblyopia treatment. It is founded on stereo vision of objects with different contrast. Fellow eye occlusion does not allow a binocular vision but only a monocular one. This approach causes brain skills loss like the perception of depth. Our system increases visual acuity for amblyopic patients using binocular vision. Binocular vision shows an object to each eye with different contrast. In fact, if we apply a right contrast ratio between amblyopic eye and lazy eye, then amblyopic patient is able to perceive both.
\end{abstract}

Keywords-Amblyopia, binocular vision, 3D technologies, contrast-based measurement, motion coherence threshold

\section{INTRODUCTION}

Amblyopia, otherwise known as lazy eye, is reduced visual acuity that results in poor or indistinct vision in an eye that is otherwise physically normal. It may exist even in the absence of any detectable organic disease. Typically amblyopia is present in only one eye and it is generally associated with a squint or unequal lenses in the prescription spectacles. This low vision is not correctable (or only partially) by glasses or contact lenses. There exist several causes of amblyopia. Anything that interferes with clear vision in either eye during the critical period (birth to 6 years of age) can result in amblyopia. The most common causes of amblyopia are constant strabismus (constant turn of one eye), anisometropia (different vision/prescriptions in each eye), and/or blockage of an eye due to trauma, lid droop, etc. If one eye sees clearly and the other sees a blur, the brain will inhibit the eye with the blur through the use of the unique good eye. The brain, for some reason, does not fully acknowledge the images seen by the amblyopic or lazy eye. Therefore, amblyopia is a neurologically active process. The inhibition process (suppression) can result in a permanent decrease of the vision in that eye which cannot be corrected with glasses, lenses, or surgery. This condition affects $2-3 \%$ of the population, which equates to conservatively around 10 million people under the age of 8 years worldwide [1].

Amblyopia is currently treated by wearing an adhesive patch over the non-amblyopic eye for several hours per day, over a period of several months. This treatment can require up to 400 hours in total to be effective [2]. This conventional patching or occlusion treatment for amblyopia often gives disappointing results for several reasons: it is unpopular, prolonged, and it can sometimes make the squint worse

NVIDIA has donated two 3D vision Kits and two QUADRO FX5800 under the professor partnership program. because it disrupts whatever fusion there is. These issues frequently results in poor or non-compliance and since the success of patching depends on compliance, it performs on average very poorly. The treatment by itself works well, but it is often abandoned because it is too much trouble to take. Very often, children are averse to wearing a patch and parents found occlusion difficult to implement [3]. For this reason, the orthoptists and ophthalmologists are continuously looking for a more acceptable solution to the problem, i.e. an effective treatment that is also complied with and so really works [4].

\section{A. Using 3D for the diagnosis and treatment of amblyopia}

In [5] we have presented a framework called $3 d 4 a m b$ for the diagnosis and treatment of amblyopia in young children. It exploits 3D technologies like the 3D Vision of NVIDIA, but it has not the aim to provide the patients with the $3 \mathrm{D}$ vision, but to allow independent binocular vision. Thus, our platform is capable to show a different image for each eye. In particular, it can show to the amblyopic eye a complete or enhanced image (from pictures, video games, and movies [6]) and to the normal eye the same image with applied a degrade filter (blur and/or less contrast and luminosity). The amblyopic eye is more stimulated while the fellow eye is penalized, but both eyes are involved in the vision process. The system is composed by a commercial personal computer, a video card with 3D capabilities, a 3D monitor and a pair of active LCD shutter glasses that the patient has to wear during the therapy. The system aims to be inexpensive (low cost, using off the shelf technologies and appliances), friendly to use (it uses simple user interfaces like joysticks), suitable for domestic use (it will use other domestic appliances like a television), easily extensible (it must be possible to easily develop new applications and programs to be added to the system), and enjoyable (it must propose recreational activities which should be appealing for young children and more compelling than classical occlusion).

\section{CONTRAST-BASED MEAUSUREMENT AND TREATMENT OF AMBLYOPIA}

\section{A. Contrast-Based Measurement of Suppression in Amblyopia}

Contrast-based measurement and treatment has been proposed in [7], [8]. It has the aim to increase the acuity of amblyopic eye through binocular vision. It is based on the following observation. If two images are shown one to each eye, the brain blends them in a unique image. This is not the 


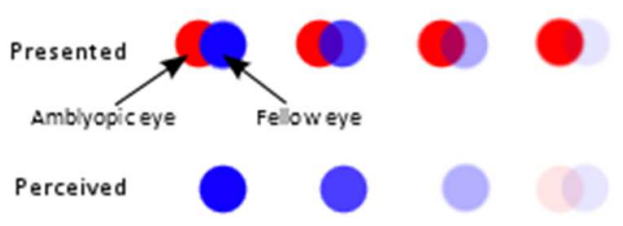

Figure 1 The image shows an example of contrastbased treatment. Blue circle is shown to fellow eye and red circle is shown to the amblyopic eye. The first row shows the images presented to each eye. Second row shows the perceived circles by amblyopic patient.

case of the amblyopic patient, where only the image shown to the fellow fixed eye will be perceived. If the information presented to the fellow eye is weakened (in this specific case with contrast reduction) the patient can stimulate the lazy eye and binocular vision can be achieved. Once binocular vision is achieved, the relative contrast or contrast imbalance between the eyes is called "balance point". At this point, suppression is no longer strong enough to inhibit binocular blending (Figure 1 ). The evaluation of the balance point is necessary for the optimal cooperation between the eyes, and for a successful treatment.

\section{B. Determining the balance point by using the dot stimulus}

The contrast balance point can be computed by exploiting the dot stimulus concept. The amblyopic patient watches visual stimulus presented dichoptically (separated images for each eye). Stimuli and noise-stimuli are represented with visible points on the screen, grouped in two sets, named signals and noise. Signals move along horizontal direction and noise moves in random directions (Figure 1).

The patient has to guess in what direction the signals are moving. The patient interaction should be minimal, he/she only should say which the right direction is. The difficulty is defined as the number of signals shown to the user when he/she chooses the correct direction. The difficulty is modified through variation of signals number, but the system keeps the number of stimuli constant.

Motion Coherence Threshold [9] is defined as the ratio between signals and total number of stimuli required by the user to identify the signal direction.

$$
M C T=\frac{\text { signal number }}{\text { stimuli number }}
$$
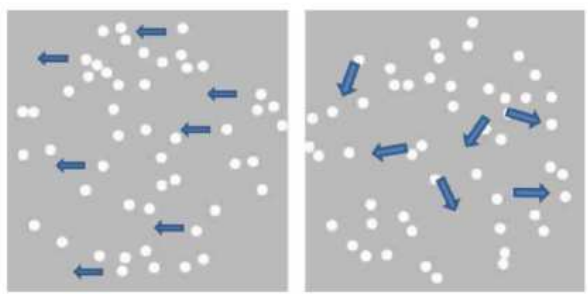

Figure 1 Signals (left picture) and noise (right picture).
A possible way to measure motion contrast threshold and balance point is presented in [8]. When MCT is given, the process has the main goal to find the balance point. The balance point represents the contrast imbalance that allows the choice of the right direction regardless from which eye receives the signals. The obtained contrast value can be used in others applications for amblyopia treatment, such as videogames. In our approach, we apply the contrast value to the game graphic elements (objects, characters, and textures) as explained in Section IV.

Note that MCT and the contrast threshold are different for each patient, so the threshold value has to be computed for every case. The contrast imbalance should decrease during each session, while patient binocular perception gets better.

\section{MOTION COHERENCE THRESHOLD EVALUATOR}

We have developed an application that allows the measurement of suppression in amblyopia and it is based on the motion coherence threshold evaluation. It exploits active 3D technology to provide patients with the dichoptic vision (as explained in Section I.A).

\section{A. Technology and treatment}

The 3D technology permits to show two different images, one for each eye. Therefore, we are able to simulate treatments based on motion coherence threshold.

We have defined a tool to perform the whole procedure. A screenshot of the application is shown in Figure 2. The doctor can set the contrast imbalance and the number of signals, while the patient has to choose the right signal direction (see Figure 2).

\section{B. MCT Evaluator: workflow for MCT computing}

The workflow of MCT evaluator is subdivided in three phases: definition of patient information, MCT evaluation, and balance point measurement.

Data capture allows obtaining of patient information, such as name, surname, age and which is his/her amblyopic eye.

Although the MCT evaluator allows the doctor to independently set MCT and contrast imbalance, we have devised the following workflow for measuring the balance point in two steps [8].

1. In the first step, our application measures the

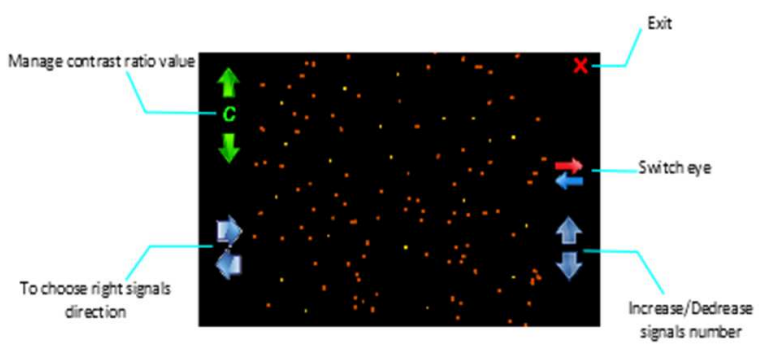

Figure 2 A screenshot of the MCT application. 
binocular motion coherence threshold, that is, the threshold number of signal dots required when both signal and noise dots are presented to both eyes at high contrast. It starts with a low signal number and increases until the patient can correctly identify the direction of the signal.

2. In the second step, the program measures the contrast imbalance required to achieve the same motion coherence threshold when the dot populations are presented to separate eyes. The signal dots are shown to the amblyopic eye, while the remaining noise dots are presented to the non-amblyopic eye at varying contrasts to assess suppression. The application starts with the worst case for an amblyopic patient: the contrast is set to zero to the non-amblyopic eye and the contrast is set to the maximum limit of the display on the amblyopic eye. This initial state simulates patching on the good eye. In other words, the amblyopic eye dominates the visual system. Then the contrast presented to the fellow eye is increased as long as the patient still chooses the right direction. The last contrast applied to the fellow eye such that the patient can still judge the right direction, is the balance point.

By this procedure, we obtain motion coherence threshold and "cr" value. $\mathrm{Cr}$ value is the contrast balance point for binocular vision of the patient.

\section{Defining variation of contrast: contrast ratio value}

The color contrast on PC screen can be expressed as brightness. Each pixel is represented by three channels (Red, Green, and Blue) and its brightness can be defined as the arithmetic average of RGB values as shown in Equation 2.

$$
\mu=\frac{R+G+B}{3}
$$

For each pixel, brightness value is modified through the parameter $\delta_{\text {bright }}$. We have defined initial RGB coordinates with $R_{i}, G_{i}, B_{i}$ and final RGB values are defined with $R_{f}, G_{f}, B_{f}$. Therefore, the final RGB values can be computed with follow formulas:

$$
\begin{gathered}
R_{f}=R_{i}+\delta_{\text {brightness }} \quad G_{f}=G_{i}+\delta_{\text {brightness }} \\
B_{f}=B_{i}+\delta_{\text {brightness }}
\end{gathered}
$$

The contrast ratio can be defined as:

$$
c r=\frac{\mu_{f}}{\mu_{i}}=\frac{R_{f}+G_{f}+B_{f}}{R_{i}+G_{i}+B_{i}}=1+3 \frac{\delta_{\text {brightness }}}{R_{i}+G_{i}+B_{i}}
$$

Contrast ratio $(c r)$ allows to increase or decrease color brightness.

\section{Important aspects of MCT Evaluator}

The application stores the final MCT value and the correct contrast ratio value. If the patient gets improvements, this outcome will be detected whether the MCT value decreases (minimal improvement acuity of amblyopic eye) or the contrast ratio decreases (maximal improvement acuity of amblyopic

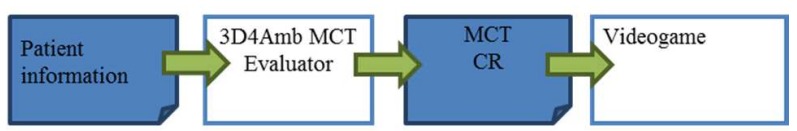

Figure 3 The picture shows the workflow from MCT evaluator for generic videogames. This software obtains patient information as input, so executes the MCT evaluator to calculate MCT value and contrast ratio.

eye). The cr value could be applied on images or videogames (Figure 3). Afterwards, we have developed a videogame to test the use of cr value. Previous mathematical model is applied to 3D models with vertex color shading (every vertex has its color).

\section{A VIDEO GAME FOR CONTRAST-BASED THERAPY}

Videogames can be considered as tools for visual skills training [10]. They promote induced training-induced learning. In particular, action videogames may be an efficient tool, as they promote brain plasticity and visual learning. These videogames require very high levels of visual attention; players are constantly monitoring the peripheral vision field; frequent, widely distributed, unpredictable events require quick and accurate aiming responses.

Action videogames can be used by amblyopic patient as alternative treatment to increase visual acuity of the lazy eye. We developed a simple action videogame with applied contrast ratio to each videogame character. The project aims to create a solid base for videogames development for future works, so we used free software according to philosophy of 3D4Amb project.

\section{A. MCT Evaluator and videogame development}

After MCT evaluation, our software application applies contrast ratio to $3 \mathrm{D}$ object of a generic videogame. The system is able to apply a contrast value to the $3 \mathrm{D}$ model that is given as input file. The output consists in two 3D models (Figure 4): the first one, with high contrast, is presented to the lazy eye, while the second one, with low contrast, is presented to the fellow eye. In this way, we separate MCT treatment from technical development of videogames. Therefore, we do not rebuild the entire videogame for each variation of contrast ratio, but we only apply contrast value to 3D models. Finally,

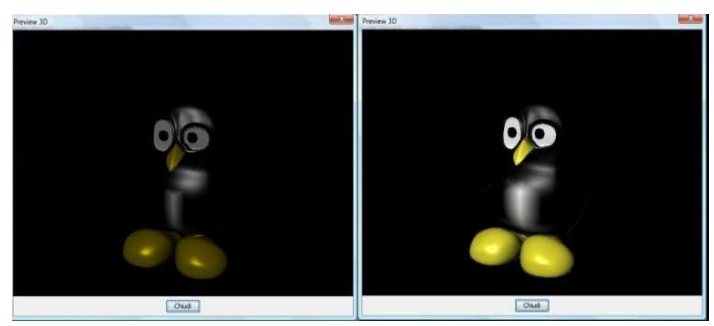

Figure 4 3D models preview with different contrast value applied. Fellow eye (left image, low contrast) and amblyopic eye (right image, high contrast). 


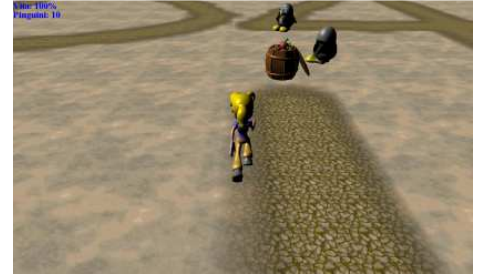

Figure 5 Screenshot of the proposed videogame Coco3D.

MCT application provides a simple way to develop videogame as tool for amblyopia treatments.

\section{B. Proposed videogame: Coco3D}

The proposed videogame is named Coco3D. It is a simple game that allows us to test how MCT application generates the 3D objects with applied contrast ratio. The game consists in a set of enemies (penguins) and a main character that has to catch all penguins without being killed (Figure 5).

\section{CONCLUSIONS}

We have presented a framework for contrast-based therapy of amblyopic patients. It exploits 3D technologies in order to allow sending different images to left and right eye. Using a simple application based on signal/noise dots we are able to measure suppression and asses the balance point in which the patient uses both eyes (binocular vision). This balance point representing the contrast imbalance between amblyopic and fellow eye, it can be used to perform treatment based on a generic video game. In the video game, models brightness is modified by using the right contrast imbalance in a way that the patient uses both eyes (rebalancing suppression in the amblyopic eye). In our approach any PC videogame using generic graphical models can be adapted to deliver the contrast-based therapy.

\section{REFERENCES}

[1] A. L. Webber and J. Wood, “Amblyopia: prevalence, natural history, functional effects and treatment," Clinical and Experimental Optometry, vol. 88, no. 6, pp. 365-375, Nov. 2005.

[2] M. Cleary, A. D. Moody, A. Buchanan, H. Stewart, and G. N. Dutton, "Assessment of a computer-based treatment for older amblyopes: the Glasgow Pilot Study.," Eye (Lond), vol. 23, no. 1, pp. 124-131, Jan. 2009.

M. Dixon-Woods, M. Awan, and I. Gottlob, "Why is compliance with occlusion therapy for amblyopia so hard? A qualitative study.,' Archives of disease in childhood, vol. 91, no. 6, pp. 491-494, Jun. 2006

[4] R. Gregson, "Why are we so bad at treating amblyopia?," Eye (London, England), vol. 16, no. 4, pp. 461-2, Jul. 2002.

A. Gargantini, "Using 3D vision for the diagnosis and treatment of amblyopia in young children," in International Conference on Health Informatics HEALTHINF 2011, 2011. (ICVR), 2011 International Conference on, 2011, pp. 1-7.
[7] L. To, B. Thompson, J. R. Blum, G. Maehara, R. F. Hess, and J. R. Cooperstock, "A Game Platform for Treatment of Amblyopia,"

IEEE Transactions on Neural Systems and Rehabilitation

Engineering, vol. 19, no. 3, pp. 280-289, Jun. 2011.

[8] J. M. Black, R. F. Hess, J. R. Cooperstock, L. To, and B.

Thompson, "The Measurement and Treatment of Suppression in Amblyopia," Journal of visualized experiments: JoVE, no. 70, 2012.

M. J. Morgan and R. Ward, "Conditions for motion flow in dynamic visual noise," Vision Research, vol. 20, no. 5, pp. 431-435, 1980.

[10] R. L. Achtman, C. S. Green, and D. Bavelier, "Video games as a tool to train visual skills," Restorative Neurology and Neuroscience, vol. 26, no. 4-5, pp. 435-446, 2008. 